\title{
Recognition models evaluated: A commentary on Keren and Baggen
}

\author{
J. E. KEITH SMITH \\ University of Michigan, Ann Arbor, Michigan 48104
}

\begin{abstract}
In a recent paper (1981), Keren and Baggen proposed two new models for alphanumeric confusion data, based on Tversky's (1977) feature model of similarity, and compared them with Luce's (1963) biased choice model. On the basis of their data, they concluded that, although the choice model fit slightly better, their models were to be preferred on grounds of parsimony and interpretability. It is shown here that both of these models are special cases of the Luce model, so that the general Luce model will necessarily fit better. This leads to considerable reinterpretation of Keren and Baggen's conclusions. Finally, better methods of estimating parameters and evaluating goodness-of-fit are suggested, taking advantage of this relation between the models.
\end{abstract}

In a recent paper, Keren and Baggen (1981) proposed two new models of alphanumeric confusion data. These models were based on the additive feature special case of Tversky's general feature model (1977). They differ from Tversky's formulation in that they make predictions about confusions rather than judged similarity.

For the basic derivation, the reader should refer to the Keren and Baggen paper. Here the more general model (Version 2) will be outlined briefly. It is assumed that the stimulus set is characterized by a known set of features $(k=1,2, \ldots, K)$. Each feature has a "salience" value, $f_{k}$, and a parameter, $a_{k}$, which measures the importance of the feature's presence in the stimulus as opposed to its presence in the contemplated response. A large $a$ implies that the presence of the feature in the stimulus "has more effect" on the response than does its absence. Finally, there is a parameter, $\theta$, measuring the importance of features common to the stimulus and the response. The dissimilarity of stimulus " $i$ " from the stimulus corresponding to response " $\mathrm{j}$ " is defined to be

$$
\mathrm{d}_{\mathrm{ij}}=\theta \sum_{A \cap B} \mathrm{f}_{\mathrm{k}}+\sum_{A \cap \bar{B}} \mathrm{f}_{\mathrm{k}} \boldsymbol{a}_{\mathrm{k}}+\sum_{\bar{A} \cap \mathrm{B}} \mathrm{f}_{\mathrm{k}},
$$

where $A$ is the set of features of the stimulus and $B$ is the set of features in the response. Next, the confusion probability, the probability of responding " $j$ " when stimulus " $i$ "' is presented is proportional to $S_{i j}$ where

$$
S_{i j}=\exp \left(-d_{i j}\right)
$$

\footnotetext{
Address correspondence to the author at Human Performance Center, University of Michigan, 330 Packard Road, Ann Arbor, Michigan 48104.
}

That is,

$$
p_{i j}=S_{i j} / \sum_{j} S_{i j}
$$

This is the more general form of the KB model, the special case being $\alpha_{k}=\alpha$ for all features.

Three important implications of this model were not discussed. First, because of the normalization carried out in Equation 3, adding a constant to each distance from stimulus " $i$ " has no effect on the predicted probabilities, since it cancels upon normalization. Thus, any feature that is common to all stimuli will have no effect on the confusion matrix. Irrelevant features should have no masking effect. Second, and equally important, the parameter sets $\left(\theta, \alpha_{k}\right)$ and $\left(\theta-c, \alpha_{k}-c\right)$ make precisely the same predictions, since

$$
\mathrm{d}_{\mathrm{ij}}=\theta \sum_{A \cap B} \mathrm{f}_{\mathrm{k}}+\sum_{A \cap \bar{B}} \alpha_{k} \mathrm{f}_{\mathrm{k}}+\sum_{\bar{A} \cap \mathrm{B}} \mathrm{f}_{\mathrm{k}}
$$

and

$$
\begin{aligned}
& d_{i j}^{*}=(\theta-c) \sum_{A \cap B} f_{k}+\sum_{A \cap \bar{B}}\left(\alpha_{k}-c\right) f_{k}+\sum_{\bar{A} \cap B} f_{k} \\
= & \theta \sum_{A \cap B} f_{k}+\sum_{A \cap \bar{B}} \alpha_{k} f_{k}+\sum_{\bar{A} \cap B} f_{k}-c \sum_{A} f_{k} \\
= & d_{i j}-c g_{i}, \\
p_{i j}^{*}= & \frac{S_{i j}^{*}}{\sum_{j} S_{i j}^{*}}=\frac{\exp \left(c g_{i}-d_{i j}\right)}{\sum_{j} \exp \left(c g_{i}-d_{i j}\right)}=\frac{\exp \left(-d_{i j}\right)}{\sum_{j} \exp \left(-d_{i j}\right)}=p_{i j} .
\end{aligned}
$$

It is therefore erroneous to interpret $\theta$ and $\alpha$ separately, since, without loss of generality, $\theta$ can arbi- 
trarily be set to (say) zero. This is technically a multicollinearity in the parameter space and is essentially impossible to detect when using a search method of estimating parameters.

The third implication is that the KB model satisfies a "cycle" condition. That is, for any three stimuli, $a, b$, and $c$, the model asserts that

$$
\mathbf{P}_{\mathrm{ab}} \mathbf{P}_{\mathrm{bc}} \mathbf{P}_{\mathrm{ca}}=\mathbf{P}_{\mathrm{ba}} \mathbf{P}_{\mathrm{ac}} \mathbf{P}_{\mathrm{cb}}
$$

or in terms of the dissimilarities:

$$
d_{a b}+d_{b c}+d_{c a}=d_{b a}+d_{a c}+d_{c b} .
$$

This implication is the one that ties the KB models to the Luce model, to which we now turn.

It is sometimes difficult to separate the Luce model itself from its extensions and interpretations. By the Luce model here is meant the assertion in Keren and Baggen's Equation 2 (p. 236), that is,

$$
S_{i j}=\beta_{j} \eta_{i j}
$$

and

$$
P_{i j}=a_{i} \beta_{j} \eta_{i j},
$$

where $a_{i}$ is a normalizing constant, equal to

$$
\left(\sum_{\mathrm{j}} \beta_{\mathrm{j}} \eta_{\mathrm{ij}}\right)^{-1}
$$

and $\eta_{\mathrm{ij}}=\eta_{\mathrm{ji}}$. Without loss of generality, one can require that

$$
\sum_{j} \beta_{j}=1
$$

and

$$
\eta_{\mathrm{ii}}=1 \quad \text { for all } \mathrm{i} .
$$

This model for square contingency tables is the same as one discussed elsewhere (Bishop, Fienberg, \& Holland, 1975; Caussinus, 1965; Smith, 1980) as the hypothesis of quasi-symmetry. It is shown that a necessary and sufficient condition for this model to hold (Bishop et al. Equation 8.2.29) is the cycle condition, that is, $\mathbf{P}_{\mathrm{ab}} \mathbf{P}_{\mathrm{bc}} \mathbf{P}_{\mathrm{ca}}=\mathbf{P}_{\mathrm{ba}} \mathbf{P}_{\mathrm{ac}} \mathbf{P}_{\mathrm{cb}}$. Sufficiency means that the KB models are themselves special cases of the Luce choice model.

The fact that the models proposed by Keren and Baggen are special cases of the Luce choice model, that is, that the expected values obtained by them fit the Luce model exactly, puts their criticisms of the choice model in quite a different light. Any data that are adequately fit by the KB models are equally well fit by a choice model. For such data, the comparison comes down to deciding whether the description provided by $\alpha$ and $\mathrm{f}$ is more intuitively appealing than that provided by $\eta$ and $\beta$. If a set of data is not fit adequately by the choice model, it also follows that it cannot be fit adequately by any feature model proposed by Keren and Baggen, since the best choice will always fit as well as or better than the best KB model.

Keren and Baggen's criticism 4 (p. 237) still holds; that is, the general choice model has a great many parameters, and if the KB feature model with a small number of features is adequate, it is parsimonious. More important, the relation between these two models suggests a rather different research strategy. For a general stimulus set, confusion data could be analyzed with a choice model. If the fit were satisfactory, examination of the resulting similarity indices would generate hypotheses about possible behaviorally significant features. If a small number of such features could be found which nearly "explained" the choice model parameters, and if further experimentation confirmed these features, a much more sophisticated theory of recognition would have been attained.

To carry out such a program, it is necessary to know the form of the specialization of the Luce model represented by the KB models. Fortunately, it is particularly simple. The derivation is presented in Appendix A. For each feature, define two parameters, $b_{k}$ and $c_{k}$ (for bias and contrast). These are defined in terms of $\alpha_{k}$ and $f_{k}$ in the KB model. It is shown that the similarity index $\eta_{\mathrm{ij}}$ is the product of the set of $c_{k}$ for the features on which stimulus " $i$ " and stimulus " $j$ " differ, and that the bias toward response " $\mathrm{j}$, " $\beta_{j}$, is proportional to the product of the set of $b_{k}$ for the features of stimulus " $j$." Thus, each cell probability can be written as a product of a set of bias and contrast factors and a stimulus normalization factor. This makes the model a log-linear model (Bishop et al., 1975), and a rather simple algorithm exists (Appendix B) for obtaining maximum likelihood estimates of the bs and cs. The Luce biasedchoice model is also log-linear, and a similar algorithm yields maximum likelihood estimates of expected cell frequencies. ${ }^{1}$ It is important that both procedures yield efficient estimators. The comparison presented by Keren and Baggen is hard to interpret, since they compare the results of a highly inefficient procedure for fitting the Luce model with a questionably efficient method of fitting their models. ${ }^{2}$

Each maximum-likelihood procedure yields a measure of goodness-of-fit, $\mathrm{G}^{2}$, the likelihood ratio statistic, which, if the corresponding model is exact, has approximately a chi-square distribution with degrees of freedom depending on the number of identifiable parameters fit. If $\mathbf{N}$ is the size of the stimulus set and $K$ is the number of features, $G^{2}$ for the more general Keren-Baggen model will have at least $N(N-1)-2 K$ degrees of freedom and the Luce $G^{2}$ will have no more than $(\mathrm{N}-1)(\mathrm{N}-2) / 2$ degrees of free- 
dom. The size of the Luce $G^{2}$ relative to its degrees of freedom is a measure of the inadequacy of any feature model of the Keren-Baggen type, however many global features are defined, and the size of the difference in $G^{2}$ relative to the difference in degrees of freedom is a measure of the necessity of the more general choice model. For instance, in the Keren and Baggen numeral example, Version 2 of their model proposes nine features, one for each of the line segments and two "global" features. Since $\theta$ is not estimable, these nine features could yield 18 parameters, an $f_{k}$ and an $a_{k}$ for each feature. In fact, because of the nature of the global features, each contributes only one additional parameter (or constraint) so Version 2 requires estimation of 16 parameters, leaving $90-16=74$ degrees of freedom for a test. The Luce model in this case has 36 degrees of freedom, although in general 1 degree of freedom is lost for every pair of stimuli that are never confused. If $\mathrm{G}^{2}$ for the Luce model is acceptably small, one would continue to entertain some form of feature model. To test whether the $\mathrm{KB}$ model is an acceptable special case, one would compute $G_{\mathrm{KB}}^{2}$ which is necessarily larger than $G_{L}^{2}$ and consider $G_{k B}^{2}-G_{L}^{2}$. This index would have 38 degrees of freedom (74-36). If this is also acceptably small, one would clearly prefer the KB model on the grounds of parsimony.

A few additional remarks on the logical status of the Keren-Baggen model are of interest. It should be clear that any global feature corresponds to a logical binary expression based on the elementary features, so 128 possible "features" exist for this type font, a smaller number if disconnected symbols are deleted. Second, there is no logical reason why the absence of a physical segment could not be defined as the presence of a feature. Thus, the "feature" that distinguishes the numeral 2 from all the rest is that it is missing line segment 6 , the vertical line segment in the lower right-hand corner of the symbol. All other numerals have this segment. It is likely that the numeral 1 is characterized perceptually by being composed of either pair of vertical line segments which form a full vertical line.
Also, it should not be supposed that the KerenBaggen model is necessarily a parsimonious special case of the choice model. If one had a feature for all but one of the characters which uniquely determines it (such as the absence of line segment 6 from numeral 2) and a feature for each pair of characters that uniquely distinguishes them from the rest (such as line segments 1 and 2 without line segment 3 , which characterizes the numerals 5 and 6 ), the KerenBaggen model would be identical with the choice model. Many other feature sets have this property, but each such set would be logically equivalent to this one. This brings up the point that even when a feature set "explains" a particular confusion table, it is not implied that this is the set being used by subjects. For any two features $f_{1}$ and $f_{2}$, one could substitute the features $f_{3}$ and $f_{4}$, where feature $f_{3}$ is the presence of both $f_{1}$ and $f_{2}$ and $f_{4}$ is the absence of both $f_{1}$ and $f_{2}$ with exactly the same fit.

\section{Data Analysis}

Luce model. The data in Keren and Baggen's Table 1 were reanalyzed using maximum likelihood methods. In terms of the Keren-Baggen measures of goodness-of-fit, the optimization criterion for the choice model was 0.0013 and the Pearson $r$ was 0.99944 . The first is smaller by a factor of 7 than that obtained by Keren and Baggen. For diagonal values alone, the indices are 0.0 and 1.0 , respectively, since it is a property of the maximum likelihood solution for the choice model that the diagonals will fit exactly. The estimated cell proportions are presented in Table 1 . Nevertheless, $\mathrm{G}^{2}$ for the choice model is 68.6 , with 36 degrees of freedom, which has attained significance of .001 . Notably large errors occur with the pairs $(1,6)$ and $(2,6)$. These four cells contribute roughly $27 \%$ of the observed $G^{2}$. Even this large a $G^{2}$ is not surprising if it is noted that the table has been combined across subjects and sessions. A somewhat more meaningful index for evaluating parsimony would be $\mathrm{G}^{2} / \mathrm{df}=1.91$.

Keren and Baggen model, Version 2. Since features 8 and 9 were not precisely defined, the compari-

Table 1

Expected Confusion Matrix by the Choice Model

\begin{tabular}{cccccccccccc}
\hline & \multicolumn{10}{c}{ Response } \\
\cline { 2 - 9 } Stimulus & 1 & 2 & 3 & 4 & 5 & 6 & 7 & 8 & 9 \\
\hline 1 & .877 & .002 & .005 & .026 & .002 & .004 & .074 & .001 & .004 & .005 \\
2 & .019 & .782 & .042 & .015 & .031 & .029 & .013 & .032 & .020 & .015 \\
3 & .031 & .034 & .681 & .006 & .027 & .003 & .040 & .029 & .134 & .014 \\
4 & .145 & .011 & .005 & .732 & .007 & .009 & .032 & .012 & .041 & .007 \\
5 & .016 & .031 & .034 & .011 & .669 & .084 & .010 & .014 & .116 & .015 \\
6 & .036 & .032 & .004 & .013 & .092 & .633 & .003 & .130 & .013 & .044 \\
7 & .255 & .005 & .021 & .019 & .004 & .001 & .667 & .004 & .009 & .016 \\
8 & .010 & .025 & .028 & .013 & .011 & .095 & .007 & .577 & .075 & .158 \\
9 & .025 & .016 & .129 & .046 & .092 & .009 & .016 & .074 & .550 & .042 \\
0 & .017 & .007 & .008 & .004 & .006 & .017 & .016 & .085 & .022 & .818 \\
\hline
\end{tabular}


son of this fit with that of Keren and Baggen may be somewhat questionable. Here, feature 8 is that which distinguishes numerals $2,3,5,7$, and 9 from the rest and feature 9 separates numerals 2,5 , and 6 . The obtained $\mathrm{G}^{2}$ is $262.2, \mathrm{G}^{2} / \mathrm{df}=3.54$. This is considerably worse than the Luce fit, even taking into account its parsimony. The difference $G^{2}$ is 193.6 with 38 degrees of freedom. The optimization criterion index is 0.011, and the Pearson $r$ is 0.995 . The optimization criterion is slightly larger than that of Keren and Baggen, just as the $G^{2}$ for their fit would be slightly larger than the one found here.

Next, a fit of the KB model was attempted using only line segment features $\left(G^{2}=387.3,76 \mathrm{df}\right)$. When feature 8 was added, $G^{2}=319.44, \mathrm{df}=75$, so feature 8 resulted in a reduction of 67.9 for only 1 degree of freedom. Adding feature 9 resulted in a further reduction of 57.2. Both are powerful features. The addition of a 10th feature distinguishes characters with enclosed areas $(6,8,9,0)$ used two parameters and resulted in a reduction of only 4.83 per degree of freedom. The principal errors remaining suggest that a feature or features discriminating between the character pairs $(2,3),(3,4),(5,7),(4,8)$, and $(8,0)$ would better describe the data.

Table 2 contains estimates of $f_{k}$ and $\alpha_{k}-\theta$ for the best fitting KB 9-feature model. The point of this table is to illustrate the fact that only 16 parameters are estimable with this form of the KB model, not 19 as claimed. Any set of values for $b_{8}, b_{9}$, the bias factors for the two global features, and $\theta$ may be entered, and will yield precisely the same distances and hence precisely the same confusion prediction as any other set.

Finally, another special case of the choice model was fit to the numeral data. It was assumed that any pair of numerals which differed by the same number of line segments should have the same similarity. This was modified by coding the pairs $(1,6)$ and $(1,9)$ both to have four distinguishing features, since it is difficult to believe that under tachistoscopic conditions a vertical line would be differently perceived whether it was on the left or the right of the figure.

Table 2

Parameter Estimates for Nine-Feature KB Model

\begin{tabular}{cll}
$\begin{array}{c}\text { Fea- } \\
\text { ture }\end{array}$ & \multicolumn{1}{c}{$\mathrm{f}_{\mathbf{k}}$} & \multicolumn{1}{c}{$\mathrm{a}_{\mathrm{k}}-\theta$} \\
\hline 1 & $\mathrm{~b}_{\mathrm{g}}+1.488$ & $\left(-\mathrm{b}_{\mathrm{g}}+.061\right) /\left(\mathrm{b}_{\mathrm{g}}+1.488\right)$ \\
2 & 1.308 & .706 \\
3 & $-\mathrm{b}_{9}+.721$ & $\left(\mathrm{~b}_{9}+.743\right) /\left(-\mathrm{b}_{9}+.721\right)$ \\
4 & 2.083 & .363 \\
5 & $-\mathrm{b}_{8}+1.163$ & $\left(\mathrm{~b}_{8}+1.121\right) /\left(-\mathrm{b}_{8}+1.163\right)$ \\
6 & $-\mathrm{b}_{8}-\mathrm{b}_{9}+.315$ & $\left(\mathrm{~b}_{8}+\mathrm{b}_{9}+.429\right) /\left(-\mathrm{b}_{8}-\mathrm{b}_{9}+.315\right)$ \\
7 & .830 & 1.606 \\
8 & $-\mathrm{b}_{8}+.732$ & $\left(\mathrm{~b}_{8}+.732\right) /\left(-\mathrm{b}_{8}+.732\right)$ \\
9 & $-\mathrm{b}_{9}+.897$ & $\left(\mathrm{~b}_{9}+.897\right) /\left(-\mathrm{b}_{9}+.897\right)$ \\
\hline
\end{tabular}

It was further assumed that response bias would be a function of the number of lines in the numeral, where features 2 and 5 or features 3 and 6 were considered to be one "line" when they both occurred. This leads to four bias parameters, one to five lines, and five similarity parameters. The attained $\mathrm{G}^{2}$, with 81 degrees of freedom, is 181.1 . This model, using seven fewer parameters, was a much better fit than the Keren-Baggen Version 2 model $\left(G^{2}=262.2\right)$. The similarity parameter estimates follow roughly the same form as shown in Keren and Baggen's Figure 1, with the similarity of numerals differing by only one segment, being roughly 4 times that for numerals differing by two segments. The other similarities were also correctly ordered. The bias parameters showed the usual preference for sparse numeral responses, with numeral 1 having a bias more than twice that of numeral 7 and the remaining biases less and roughly the same.

Of course, this model too is clearly inadequate by conventional tests. Its major failings are instructive. The first is that it significantly underestimates the similarity of the numerals 2 and 5 and the bias toward response " 0. " The similarity between 2 and 5 corresponds to a 2-feature difference, and the bias toward " 0 " is the same as that toward 7.

\section{Summary}

Tversky's feature model for judged similarities when appropriately modified to apply in a recognition paradigm would be an interesting competitor for the Luce biased-choice model. Keren and Baggen attempted this modification in a sensible and straightforward way, but ended up with a special case of the choice model. It is clear that a competitive version will either need to avoid the additive features assumption or the negative exponential conversion of distances to similarities.

The models proposed by Keren and Baggen do, however, suggest ways of specializing the choice model to have fewer parameters and a more processoriented interpretation. They provide a competitive alternative to the geometrical method of converting similarities to distances and searching for a lowdimensional solution. It should be possible to use the biased choice solution to suggest likely features and to search for a minimal set of features to "explain" the similarity structure.

To do this, an efficient method of estimation of parameters is needed, and the maximum likelihood method is described.

These methods have been illustrated using the data published by Keren and Baggen on tachistoscopic numeral recognition. The biased-choice model, taking into account the fact that the presented data have been averaged across subjects and sessions, provides a reasonable account, but the set of features pro- 
posed by Keren and Baggen are clearly insufficient, even allowing for the small number of parameters estimated.

\section{REFERENCES}

Bishop, Y. M. M., Fienderg, S. E., \& Holland, P. W. Discrete multivariate analysis. Cambridge, Mass: M.I.T. Press, 1975.

Caussinus, H. Contribution a l'analyse statistique des tableaus de correlation. Ann. Fac. Sci. Univ. Toulouse, 1966, 29, 77. 182.

KeneN, G., \& BagGeN, S. Recognition models of alphanumeric characters. Perception \& Psychophysics, 1981, 29, 234-245.

Luce, R. D. Detection and recognition. In R. D. Luce, R. R. Bush, \& S. E. Galanter (Eds.), Handbook of mathematical psychology (Vol. 1). New York: Wiley, 1963.

SmITH, J. E. K. Models of identification. In R. Nickerson (Ed.), Attention and performance VIII. Hillsdale, N.J: Erlbaum, 1980.

Tvensky, A. Features of similarity, Psychological Review, 1977, $84,327-352$.

\section{NOTES}

1. Once maximum likelihood estimators of cell frequencies, and hence cell probabilities, are available, any consistent estimators of $\eta$ and $\beta$ applied to these expected frequencies yield maximum likelihood estimates of the parameters. Keren and Baggen's Equation 3 is a convenient way of calculating similarity estimates. Their Equation 4 is incorrect. An equation that provides a consistent estimator and a computing formula when applied to expected $\mathrm{p}_{\mathrm{ij}} \mathrm{s}$

$$
\hat{\beta}_{j}=1 / \sum_{k=1}^{N}\left(p_{j k} p_{k k} / p_{k j} p_{j j}\right)^{1 / 2}
$$

2. The major criticism of the Keren-Baggen estimation procedure is of their choice of an optimality criterion. While it may be "customary," it has the defect of considering only the absolute value of the deviation between observed and expected values. Thus a difference of 10 observations is as damaging to the criterion whether it arises as a difference between 10 and 20 or a difference between 250 and 260 . Such a criterion would be appropriate if cell frequencies were assumed to have constant variance, but for contingency tables, a criterion such as chi-square or the likelihood ratio index is to be preferred.

\section{APPENDIX A}

\section{Representation of the KB Model as a Choice Model}

From Keren and Baggen's Equation 5, and later discussion,

$$
d_{a b}=\theta f(A \cap B)+\alpha f(A-B)+f(B-A),
$$

where $f$ is an additive set function. If we define indicator functions

$$
\delta_{i k}=\begin{aligned}
& 1 \text { if feature } k \text { is a feature of stimulus } i \\
& 0 \text { otherwise. }
\end{aligned}
$$

Equation 9 can be rewritten as

$$
d_{i j}=\sum_{k} f_{k}\left[\theta \delta_{i k} \delta_{j k}+\alpha_{k} \delta_{i k}\left(1-\delta_{j k}\right)+\left(1-\delta_{i k}\right) \delta_{j k}\right],
$$

where $\alpha$ has been generalized to depend on the particular feature. Expanding the large factor,

$$
d_{i j}=\sum_{k} \alpha_{k} f_{k} \delta_{i k}+\sum_{k} f_{k} \delta_{j k}+\sum_{k} f_{k} \delta_{i k} \delta_{j k}\left(\theta-\alpha_{k}-1\right)
$$

The first term on the right can be omitted because it will cancel out of Keren and Baggen's Equation 7. The second term is a function only of the response and corresponds to the bias factor in the choice model, and the third term is a symmetric function of the stimulus and response and corresponds to the similarity factor in the choice model. To simplify the final expression, using the simple identity in indicator variables,

$$
\delta_{i k} \delta_{j k}=\left[\delta_{i k}+\delta_{j k}-\left(\delta_{i k}-\delta_{j k}\right)^{2}\right] / 2,
$$

and

$$
\begin{aligned}
\mathrm{d}_{\mathrm{ij}}= & \sum_{\mathbf{k}} \delta_{i k} \mathrm{f}_{\mathrm{k}} \frac{\theta+\alpha_{\mathbf{k}}-1}{2}+\sum_{\mathbf{k}} \delta_{\mathrm{jk}} \mathrm{f}_{\mathrm{k}} \frac{\theta-\alpha_{\mathrm{k}}+1}{2} \\
& -\sum_{\mathbf{k}}\left(\delta_{\mathrm{ik}}-\delta_{\mathrm{jk}}\right)^{2} \mathrm{f}_{\mathbf{k}} \frac{\theta-\alpha_{\mathbf{k}}-1}{2}
\end{aligned}
$$

Again dropping the first term which will cancel in KB Equation 7, and defining $b_{k}$, a feature bias term, and $c_{k}$, a feature contrast term,

$$
\begin{aligned}
b_{k} & =\exp \left[-f_{k}\left(\theta-a_{k}+1\right) / 2\right] \\
c_{k} & =\exp \left[f_{k}\left(\theta-a_{k}-1\right) / 2\right]
\end{aligned}
$$

we get

$$
d_{i j}^{\prime}=-\sum_{k} \delta_{j k} \ln \left(b_{k}\right)-\sum_{k}\left(d_{i k}-\delta_{j k}\right)^{2} \ln \left(c_{k}\right)
$$

and

$$
S_{i j}=\prod_{k} b_{k}^{\delta_{j k}} \prod_{k} c_{k}^{\left(\delta_{i k}-\delta_{j k}\right)^{2}}
$$

Defining

$$
a_{i}^{-1}=\sum_{j} S_{i j}
$$

and applying the KB Equation 7 ,

$$
\mathrm{p}_{\mathrm{ij}}=\mathrm{a}_{\mathrm{i}}\left(\prod_{\mathrm{k}} \mathrm{b}_{\mathrm{k}}^{\delta_{\mathrm{jk}}}\right)\left(\prod_{\mathrm{k}}^{\left(\delta_{\mathrm{ik}}-\delta_{\mathrm{jk}}\right)^{2}}\right)
$$

or

$$
\mathrm{p}_{\mathrm{ij}}=\mathrm{a}_{\mathrm{i}} \beta_{\mathrm{j}} \eta_{\mathrm{ij}},
$$

the form of a quasi-symmetric model, where

$$
\dot{\eta}_{i j}=\prod_{k} c_{k}^{\left(d_{i k}-\delta_{j k}\right)^{2}},
$$




$$
\beta_{\mathrm{j}}=\prod_{\mathbf{k}} \mathbf{b}_{\mathbf{k}}^{\delta_{\mathrm{jk}}}
$$

and

$$
a_{i}=\left(\sum_{j} \beta_{i} \eta_{i j}\right)^{-1}
$$

we have

$$
p_{i j}=a_{i} \beta_{j} \eta_{i j},
$$

the choice model. Inverting Equation 14, we have

$$
\begin{gathered}
f_{k}=\ln b_{k}+\ln c_{k} \\
\left(a_{k}-\theta\right) f_{k}=\ln b_{k}-\ln c_{k} .
\end{gathered}
$$

Note that $\eta$ is a product of factors, one for each feature that distinguishes between the symbols, while $\beta$ is a product of factors, one for each feature of the response.

\section{APPENDIX B}

\section{Maximum Likellhood Estimates}

In both the general Luce (1963) model and the special case (Version 2) proposed by Keren and Baggen (1981), each cell probability in the model is written as a product of factors characteristic of that cell, one factor for the row plus one or more others depending on the cell identity. Thus, the logarithm of the cell probability (or, as will be more useful, the logarithm of the cell frequency) can be written as a linear combination of numbers, each with a coefficient of one or zero, depending on whether the factor is characteristic of the cell or not.

This is the simplest type of log-linear model, and it is easily shown (Bishop, Fienberg, \& Holland, 1975) that for each factor the sum of the maximum likelihood estimates of the cell frequencies, $m_{i j}$, of cells having the factor is equal to the same sum of observed frequencies, $n_{i j}$, and the same also holds for cells not having the factor. Each factor divides the cells into two subjects, and the sum of expected frequencies for each subset must equal the sum of observed frequencies for that subset. If a set of factors partitions the cells into more than two groups, each group satisfies the same property.
Table 3

One Cycle of Fitting a Choice Model

\begin{tabular}{rrrrrrrrr}
\hline & A & B & C & & A & B & C \\
\hline & \multicolumn{1}{c}{ (a) Data } & & \multicolumn{2}{c}{ (d) Adjusted for Column } & Factors \\
A & 30 & 10 & 8 & A & 13.56 & 15.46 & 18.98 \\
B & 14 & 40 & 12 & B & 18.64 & 21.25 & 26.10 \\
C & 6 & 7 & 50 & C & 17.80 & 20.29 & 24.92
\end{tabular}

(b) Initial $\mathrm{m}_{\mathbf{i j}}$

$\begin{array}{rrrrrrrr}\text { A } & 1 & 1 & 1 & \text { A } & 30.00 & 10.88 & 7.22 \\ \text { B } & 1 & 1 & 1 & \text { B } & 13.12 & 40.00 & 10.69 \\ \text { C } & 1 & 1 & 1 & \text { C } & 6.78 & 8.31 & 50.00\end{array}$

(c) Adjusted for Row Factors

(f) Maximum

\begin{tabular}{lllllrrr} 
A & 16 & 16 & 16 & A & 30.00 & 10.12 & 7.88 \\
B & 22 & 22 & 22 & B & 13.88 & 40.00 & 12.12 \\
C & 21 & 21 & 21 & C & 6.12 & 6.88 & 50.00 \\
\hline
\end{tabular}

It has been proved that there exists only one solution, and a convergent algorithm (not a search algorithm) exists for finding it. The algorithm consists of starting with a set of $m_{i j}$ which fit the model vacuously, usually $m_{i j}=1$ for all $\mathrm{i}, \mathrm{j}$. Then, for each factor in turn, the $\mathrm{m}_{\mathrm{ij}}$ are multiplicatively adjusted to satisfy the summation condition. This cycle continues until the last cycle has no appreciable effect, that is, all multipliers are effectively 1 . Given below are examples.

\section{General Choice Model}

In this model there are three types, or sets, of factors, row factors, column factors, and similarity factors. The row factors imply that $\mathrm{m}_{\mathrm{i}+}=\mathrm{n}_{\mathrm{i}+}$, the column, or bias, factors imply that $m_{+j}=n_{+j}$, and the similarity factors imply that $m_{i j}+m_{j i}=n_{i j}+n_{j i}$. Note that this also implies that $m_{i i}=n_{i i}$, where $m_{i+}$ and $n_{i+}$ are the sums of the observed and expected frequencies for the $\mathrm{i}^{\text {th }}$ row, and $m_{+j}$ and $n_{+j}$ are the sums of the observed and expected frequencies for the jth column. One cycle of the process is illustrated in Table 3.

\section{Keren-Baggen Model, Version 2}

Using the data in Table 3, suppose there were two features, feature 1 possessed by $A$ and $B$ and feature 2 possessed by $A$ and $C$. A cycle of computations is presented in Table 4. The KB model estimates four parameters.

\begin{tabular}{|c|c|c|c|c|c|c|c|c|c|c|c|c|c|c|c|}
\hline & $\mathbf{A}$ & B & $\mathrm{C}$ & & $\mathbf{A}$ & $\mathbf{B}$ & $\mathrm{C}$ & & $\mathbf{A}$ & B & $\mathbf{C}$ & & A & B & $\mathrm{C}$ \\
\hline \multicolumn{4}{|c|}{ (a) Initial $\mathrm{m}_{\mathbf{i j}}$} & \multicolumn{4}{|c|}{ (c) Adjusting for $b_{1}$} & \multicolumn{4}{|c|}{ (e) Adjusting for $b_{2}$} & \multicolumn{4}{|c|}{ (g) MLE } \\
\hline $\begin{array}{l}\text { A } \\
\text { B } \\
\text { C }\end{array}$ & $\begin{array}{l}1 \\
1 \\
1\end{array}$ & $\begin{array}{l}1 \\
1 \\
1\end{array}$ & $\begin{array}{l}1 \\
1 \\
1\end{array}$ & $\begin{array}{l}\text { A } \\
\text { B } \\
\text { C }\end{array}$ & $\begin{array}{l}14.51 \\
19.95 \\
19.04\end{array}$ & $\begin{array}{l}14.51 \\
19.95 \\
19.04\end{array}$ & $\begin{array}{l}18.98 \\
26.10 \\
24.92\end{array}$ & $\begin{array}{l}\text { A } \\
\text { B } \\
\text { C }\end{array}$ & $\begin{array}{r}22.93 \\
31.51 \\
7.78\end{array}$ & $\begin{array}{r}21.00 \\
28.87 \\
7.13\end{array}$ & $\begin{array}{r}7.75 \\
10.66 \\
39.37\end{array}$ & $\begin{array}{l}\text { A } \\
\text { B } \\
\text { C }\end{array}$ & $\begin{array}{r}23.92 \\
17.54 \\
8.54\end{array}$ & $\begin{array}{r}12.54 \\
40.00 \\
4.46\end{array}$ & $\begin{array}{r}11.54 \\
8.46 \\
50.00\end{array}$ \\
\hline \multicolumn{4}{|c|}{ (b) Adjusting for Rows } & \multicolumn{4}{|c|}{ (d) Adjusting for $c_{1}$} & \multicolumn{4}{|c|}{ (f) Adjusting for $c_{2}$} & & & & \\
\hline $\begin{array}{l}\text { A } \\
\text { B } \\
\text { C }\end{array}$ & $\begin{array}{l}16 \\
22 \\
21\end{array}$ & $\begin{array}{l}16 \\
22 \\
21\end{array}$ & $\begin{array}{l}16 \\
22 \\
21\end{array}$ & $\begin{array}{l}\text { A } \\
\text { B } \\
\text { C }\end{array}$ & $\begin{array}{r}22.27 \\
30.61 \\
7.56\end{array}$ & $\begin{array}{r}22.27 \\
30.61 \\
7.56\end{array}$ & $\begin{array}{r}7.53 \\
10.36 \\
38.24\end{array}$ & $\begin{array}{l}\text { A } \\
\text { B } \\
\text { C }\end{array}$ & $\begin{array}{r}28.80 \\
19.27 \\
9.77\end{array}$ & $\begin{array}{r}12.84 \\
36.26 \\
4.36\end{array}$ & $\begin{array}{r}9.73 \\
6.52 \\
49.44\end{array}$ & & & & \\
\hline
\end{tabular}
While the choice model estimates five, $\mathrm{G}^{2}$ for the $\mathrm{KB}$ model is 7.35 with 2 degrees of freedom and $\mathrm{G}^{2}$ for the choice

Table 4

One Cycle of Fitting a Feature Model

Note-MLE $=$ maximum likelihood estimate. 
model is 0.010 . The difference, with 1 degree of freedom, is 7.34 and would be highly significant. So, for this madeup data, the choice model fits exceptionally and the twofeature KB model does not. Adding a feature that is shared by $B$ and $C$ would make the two models identical.

\section{Keren-Baggen Model, Version 1}

Fitting Version 1 of the feature model with maximum likelihood methods is rather more difficult, and will not be discussed here. For any particular fixed $\alpha$, a maximum likelihood solution can be found for the $f_{k}$. Then a search over the range of $\alpha$ must be carried out to solve for its estimate. It would normally not be carried out unless the Version 2 solution fit satisfactorily and the estimated $\alpha_{\mathbf{k}}$ seemed to be fairly similar. For the data at hand, neither condition is met.

(Manuscript received August 4, 1981;

revision accepted for publication October 14, 1981.) 